\title{
Nanostructure Analysis of Diamond Cold-Cathode Field Emitters
}

\author{
T. C. Wade, ${ }^{*}$ J. E. Wittig, ${ }^{* *}$ J. L. Davidson, ${ }^{* *}$ W.P. Kang, ${ }^{* *}$ N. Tolk*** and L.F. Allard**** \\ * Interdisciplinary Material Science \& Eng., Vanderbilt University, Nashville, TN 37235 \\ ** Electrical Eng. \& Computer Sci., Vanderbilt University, Nashville, TN 37235 \\ *** Physics \& Astronomy, Vanderbilt University, Nashville, TN 37235 \\ **** Materials Science \& Technology Div., Oak Ridge National Laboratory, Oak Ridge TN 37831
}

Chemical-vapor-deposited (CVD) diamond is an attractive material for electron field emitters because of its low or negative electron affinity, mechanical strength, and chemical inertness [1]. Nanocrystalline diamond possesses unique properties including controlled $\mathrm{sp}^{2}$-carbon content and $\mathrm{p}$ type electrical conductivity with boron doping. Arrays of ultra-sharp diamond tips with a radius of curvature less than $5 \mathrm{~nm}$ have been fabricated and show significant improvement in emission brightness and turn-on field compared to conventional field emitters [2]. Irregularities in emission behavior between tips were historically attributed to anomalies in the fabrication process: "sharp" or "less sharp" tips. However, we observe large differences in electron emission thresholds between tips that appear to be equally well formed. This study examines the emitter's sub-surface structure to provide insight into how surface and subsurface structure may affect emission.

The diamond field emitter tips were produced by using photolithographic-patterned pyramidal etchpits on a silicon substrate as a mold for the plasma-assisted CVD diamond growth. Details of the growth process are discussed in earlier papers $[3,4]$. Cross-sectional TEM samples were prepared by standard FIB lift-out techniques with the additional step of using a layer of electron-beam-deposited Pt before the ion-beam Pt deposition, in order to preserve the $5 \mathrm{~nm}$ radius of curvature field emission tips. To image the actual field emission tip in cross-section, it is critical to thin the FIB lamella equally from each side. Bright-field (BF) STEM images of the emitter tip cross sections were recorded at $200 \mathrm{kV}$ with a sub-0.1-nm probe in a probe-corrected JEOL 2200FS STEM/TEM. Figure 1 shows an example where the sharp emitter tip (observed in the SEM image insert) was removed during final FIB thinning. However, Fig. 2 reveals that with careful sample preparation technique, it is possible to capture the emitter tip in the cross-sectional sample.

Low magnification BF-STEM images of the cross-sectioned pyramidal tips in Figs.1-2 illustrate the two-step fabrication process in which 1-2 $\mu \mathrm{m}$ of nanodiamond is grown to form the emitter tip surface followed by $\sim 10 \mu \mathrm{m}$ of microdiamond that produces the bulk of the tip as well as the support layer. The core of the emitter consists of electrically conductive boron-doped microcrystalline diamond shown in Fig. 3 with characteristic diamond cubic twinning. The graphite layers in the image are likely specimen preparation artifacts, although the presence of graphitic material in these CVD diamond emitter tips is still an open question. Figure 4 is a BF image of the nanocrystalline diamond layer from an area near the surface of Fig. 1. Crystallites on the order of $5 \mathrm{~nm}$ in size are evident in this image. However, the image quality is limited by the fact that the FIB sample is still too thick to take full advantage of the resolution possible with an aberration-corrected STEM. Final thinning using a nanomill (Fischione Co.) with low energy Ar may be the solution for removing any surface artifacts and reducing the sample thickness. Comparison of the nanostructure of emitter tips with variation in field emission characteristics will provide insight into the influence of the nanocrystalline structure on the electronic properties [5]. 
References

1. Van Der Weide, J., et al., Physical Review B, 1994. 50(8): p. 5803.

2. $\quad$ Alves, M.A.R., et al., Microelectronic Engineering, 2004. 75(4): p. 383-388.

3. Wisitsora-at, A., et al., Applied Surface Science, 1999. 146(1-4): p. 280-286.

4. Subramanian, K., et al., Diamond and Related Materials, 2006. 15(4-8): p. 1126-1131.

5. Thanks to Dr. Steven Prawer, Igor Aharonovich, and Dr. Sergey Rubanov at the University of Melbourne for assistance with TEM sample preparation. Research at the Oak Ridge National Laboratory SHaRE User Facility was supported by the Scientific User Facilities Division, Office of Basic Energy Sciences, U.S. Department of Energy. This work was funded by the US Department of Defense Extreme Light Source program.

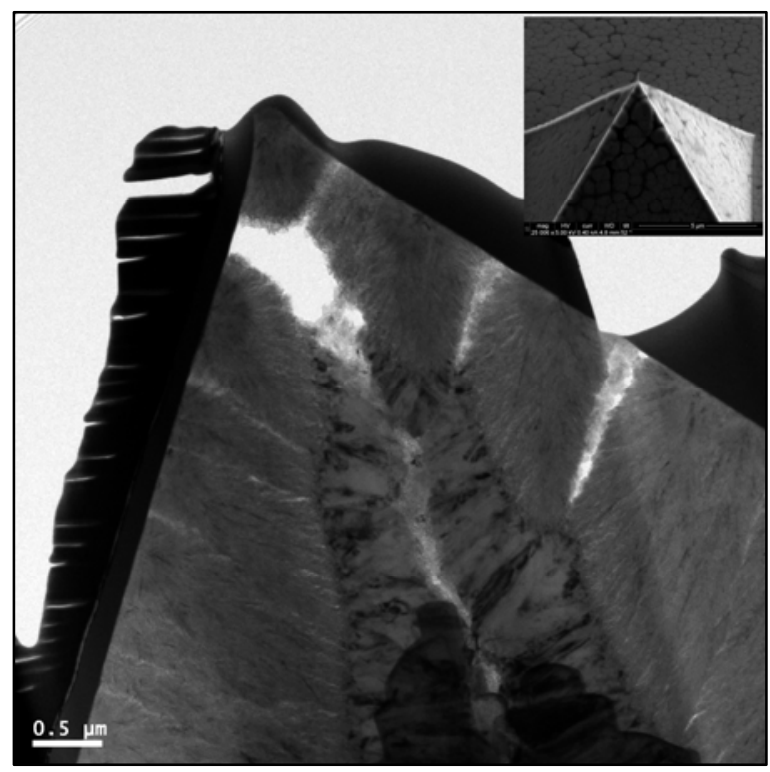

FIG. 1. Bright Field image of emitter tip.

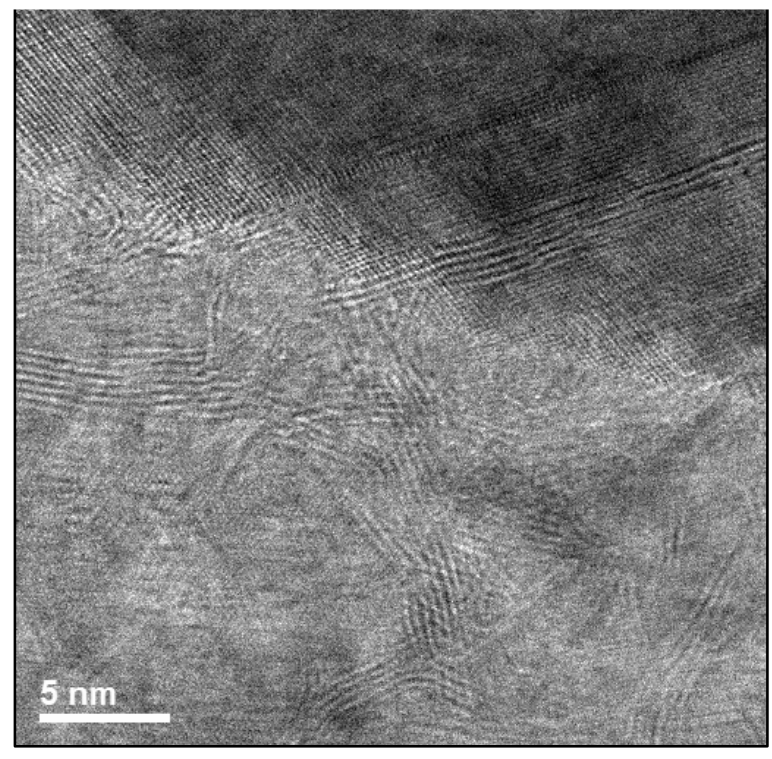

FIG. 3. BF image of microcrystalline diamond.

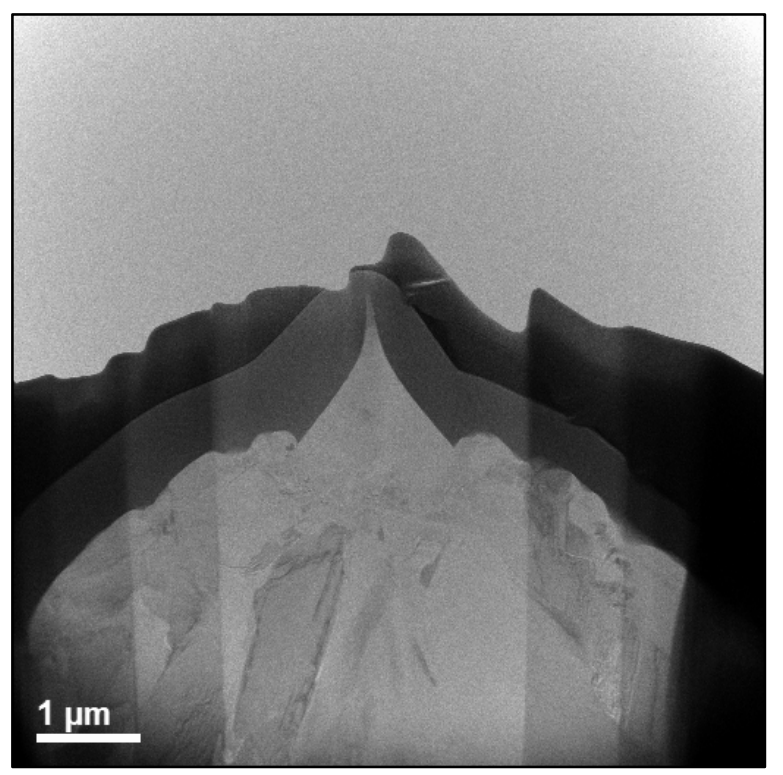

FIG. 2. Bright Field image of emitter tip.

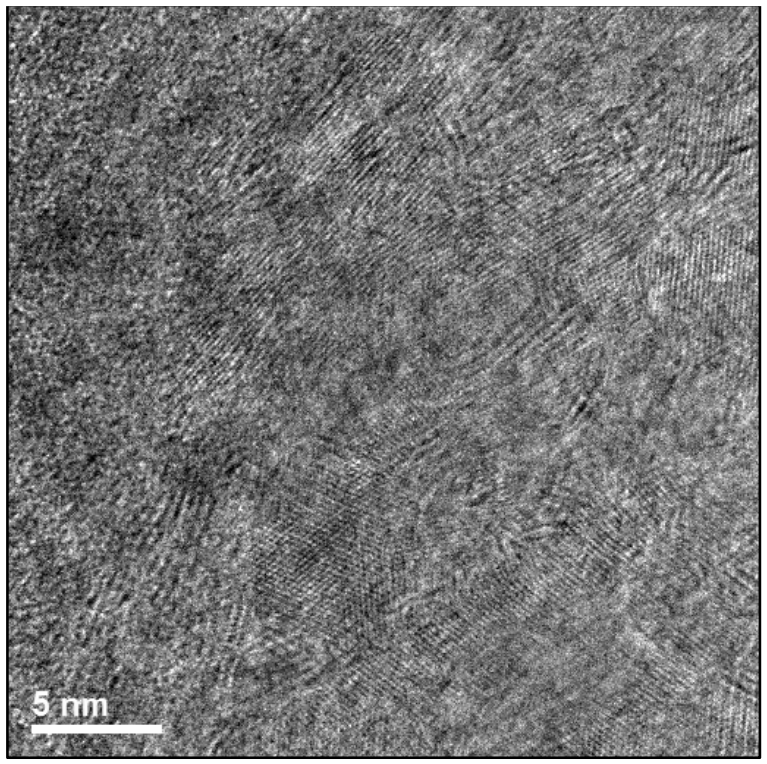

FIG. 4. BF image of nanocrystalline diamond. 